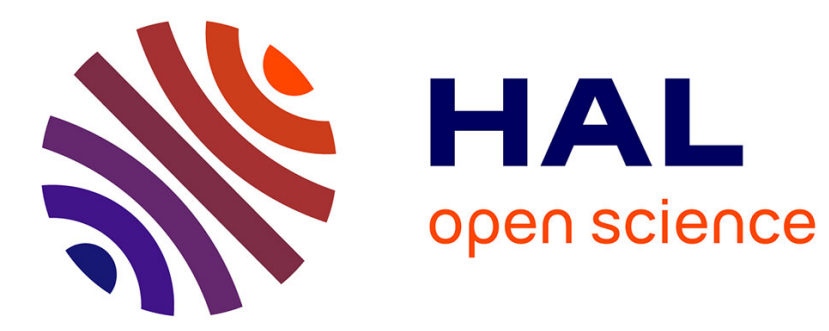

\title{
Raison statistique et raison sociologique chez Maurice Halbwachs
}

Olivier Martin

\section{To cite this version:}

Olivier Martin. Raison statistique et raison sociologique chez Maurice Halbwachs. Revue d'histoire des sciences humaines, 1999, p.69-101. 10.3917/rhsh.001.0069 . hal-01261273

\section{HAL Id: hal-01261273 \\ https://hal.science/hal-01261273}

Submitted on 25 Jan 2016

HAL is a multi-disciplinary open access archive for the deposit and dissemination of scientific research documents, whether they are published or not. The documents may come from teaching and research institutions in France or abroad, or from public or private research centers.
L'archive ouverte pluridisciplinaire HAL, est destinée au dépôt et à la diffusion de documents scientifiques de niveau recherche, publiés ou non, émanant des établissements d'enseignement et de recherche français ou étrangers, des laboratoires publics ou privés. 


\title{
Raison statistique et raison sociologique chez Maurice Halbwachs
}

\author{
Olivier MARTIN
}

\section{Résumé}

Maurice Halbwachs est connu pour être, parmi les sociologues durkheimiens, celui qui a porté le plus grand intérêt à la statistique et aux mathématiques, à leurs usages et à leurs limites en sociologie. Elles constituent pour lui un instrument privilégié pour bâtir une sociologie positive mais en même temps le pouvoir réel qu'il leur attribue est très limité : elles ne peuvent servir qu'à établir des faits que le sociologue doit ensuite interpréter et expliquer, et qui doivent être replacés dans leur contexte social précis. Il rejette l'abstraction mathématique et les abus de la standardisation. Au fond, sa position vis-à-vis des statistiques est ambivalente : il souhaite faire appel à la statistique pour étudier les faits sociaux, sans réduire ces faits à de simples collections d'événements ou de comportements individuels.

Mots-clefs : Démographie - Économie - Sociologie - Statistique Probabilité - Mathématique - Méthodologie - Halbwachs - Quetelet

Summary: Statistical thinking and sociological thinking in Maurice Halbwachs' work

Maurice Halbwachs is known as the durkheimian sociologist who took the greatest interest in statistics and mathematics, and in their use - and the limits thereof - in sociology. He viewed statistics as the best tool for the development of positive sociology. But at the same time, he attributed very limited actual power to statistics and mathematics, which could only be used to establish facts. It is then up to the sociologist to interpret and explain those facts, which need to be situated in their exact social context. He rejected mathematical abstraction and the abuse of standardisation. His attitude toward statistics is basically ambivalent, in that he wished to apply it to the study of social facts without reducing those facts to mere collections of events or of individual acts.

Key-words : Demography - Economics - Sociology - Statistics Probability - Mathematics - Methodology - Halbwachs -Quetelet 
La chose est entendue : Maurice Halbwachs est, parmi les héritiers ou continuateurs d'Émile Durkheim celui qui porte le plus grand intérêt à la statistique et à son usage en sciences sociales : en économie, en démographie et bien entendu en sociologie ${ }^{1}$. Il est proche, en cela notamment, d'un autre membre du cercle durkheimien, François Simiand, spécialiste de sociologie économique, concevant la statistique comme moyen d'observation et d'expérimentation ${ }^{2}$. Les deux hommes se sont rencontrés au sein du Groupe de l'unité socialiste, fondé en 1899 par Lucien Herr, actif bibliothécaire de l'École Normale Supérieure de la rue d'Ulm. C'est par l'intermédiaire de Simiand qu'Halbwachs rejoint l'équipe de l'Année Sociologique en $1905^{3}$. En 1936, Halbwachs consacre un article théorique à la pensée méthodologique de Simiand, récemment et brutalement décédé ${ }^{4}$. Toutefois, tout en étant largement influencé par Simiand, tout en reconnaissant sa dette à son "grand $\mathrm{ami}^{5}{ }^{5}$, Halbwachs poursuit une réflexion personnelle sur la statistique et l'utilise sur des terrains plus variés que Simiand.

Évoquant la période où Maurice Halbwachs s'oriente vers la sociologie en abandonnant la philosophie et la métaphysique qui constituaient les matières de sa formation initiale, sa sœur Jeanne Alexandre précise que « redevenant étudiant, il fit son droit, apprit l'économie politique, s'exerça aux mathématiques ${ }^{6} »$. Son intérêt pour les mathématiques lui est donc venu au moment de sa réorientation vers les sciences sociales. Et si nous pouvons facilement imaginer qu'il n'a jamais envisagé de devenir mathématicien ou statisticien, il paraît en revanche certain que, dès l'origine de cette réorientation, notamment en raison de l'influence exercée par Simiand, Halbwachs a porté un regard attentif à la question du rôle que peuvent jouer statistiques et mathématiques en sociologie. Cette attention ne se démentira jamais : même s'ils ne résument pas l'ensemble de son œuvre à eux seuls, les écrits où il aborde la question des statistiques, leurs places et rôles en sociologie, parsèment l'ensemble de sa bibliographie. Évoquons rapidement son œuvre et son parcours au prisme de ses intérêts et usages de la statistique.

\footnotetext{
${ }^{1}$ Sur les rapports de Halbwachs à l'économie, voir notamment l'article de STEINER dans le même numéro et PFEFFERKORN, 1997 ; sur ses rapports à la démographie voir LENOIR, 1997. Sur la place générale de Halbwachs parmi les sociologues durkheimiens, voir BESNARD, 1979 et MARCEL, 1997. Quelques pistes pour comprendre Halbwachs statisticien ont déjà été ouvertes dans VerRet, 1972, LENOIR, 1997 ou BAUDELOT, ESTABLET, 1994 et dans quelques écrits abordant de façon générale l'histoire des rapports de la sociologie à la statistique, en particulier DESROSIÈRES (1982, 1991, 1993). Nous entendons approfondir ces différentes pistes et éclairer des aspects nouveaux, afin de saisir la pensée statistique d'Halbwachs dans son ensemble, dans sa cohérence et dans ses limites.

${ }^{2}$ SiMIAND, 1922 et 1987.

${ }^{3}$ BeSNARD, 1979, 18 ; MARCEL, 1997, 119, 199.

${ }^{4}$ HALBWACHS, 1936a.

${ }^{5}$ HALBWACHS, 1944 (1935), 113.

${ }^{6}$ AlEXANDRE, 1940-1948, 4.
} 
Les données statistiques et leur traitement sont au cœur de plusieurs des ouvrages majeurs d'Halbwachs. Sa thèse, publiée en 1912 sous le titre $L a$ classe ouvrière et les niveaux de vie, s'appuie de façon déterminante sur l'analyse de données statistiques allemandes (produites par l'Union des travailleurs des métaux et par l'Office impérial de statistique en 1909) ${ }^{7}$. Fondée sur l'observation directe des faits étudiés et sur le traitement statistique de ces faits, sa recherche est certainement la première thèse de ce type soutenue dans une université française. Vingt ans plus tard, il réalise une étude plus étendue sur le même sujet, L'évolution des besoins dans les classes ouvrières (1933), en s'appuyant sur des données et des enquêtes d'origines diverses (Bureau International du Travail, enquêtes françaises, allemandes, anglaises et américaines). Et sa recherche sur les Causes $d u$ suicide (1930), qui actualise et corrige l'ouvrage de Durkheim sur le même sujet (1897), repose également sur des analyses de données statistiques variées: données d'état civil, procès-verbaux des médecins ou policiers, enregistrements de l'administration judiciaire, annuaires... À ces ouvrages majeurs, nous pouvons ajouter nombre d'articles, de rapports ou de comptes rendus abordant la question du rôle des statistiques en sciences sociales : bien que le dénombrement soit une opération délicate, il est néanmoins possible d'estimer à plus de 35 le nombre d'articles ou de comptes rendus abordant principalement ou exclusivement la question de la statistique en sciences sociales, ou utilisant de façon dominante des données statistiques ${ }^{8}$. Collaborateur régulier de l'Année sociologique, des Annales d'histoire économique et sociale, du Journal de la société statistique de Paris, Halbwachs y publie fréquemment, notamment durant la période 1905-1930, des comptes rendus d'ouvrages de statistique. En particulier, il rédige, pour l'Année sociologique dont il est devenu un collaborateur régulier en 1905 puis pour les Annales sociologiques, de nombreuses notes bibliographiques concernant les entrées « Statistique morale » et «Statistique».

Si ses ouvrages sur les budgets ouvriers et sur le suicide sont les traces les plus visibles de son usage des données statistiques, il existe plusieurs symboles de sa réflexion permanente, souvent épistémologique, sur les outils statistiques. Le premier de ces signes est certainement la thèse (secondaire) qu'il consacre à une analyse de la théorie de l'homme moyen de Quetelet en 1912 : il conduit une critique argumentée de l'usage de la notion de moyenne et de la théorie des erreurs chez le savant belge.

Le symbole suivant est un livre, issu d'une collaboration nouée à Strasbourg par Halbwachs. Professeur titulaire d'une chaire de sociologie à la Faculté des Lettres de Strasbourg (une des deux seules chaires de sociologie à

\footnotetext{
${ }^{7}$ HaLBWACHS, $1912 \mathrm{~b}, 136$ sqq.

${ }^{8}$ Décompte réalisé à partir de la bibliographie constituée par Victor Karady et publiée dans HALBWACHS, 1972.
} 
l'époque) de 1919 à 1935, il côtoie au sein de l'université Maurice Fréchet et Georges Cerf, professeurs de mathématiques à la Faculté des Sciences. Futur membre de l'Académie des Sciences, spécialiste de calcul des probabilités et d'analyse fonctionnelle arrivé à Strasbourg en 1920, Maurice Fréchet (18781973) dispense avec Halbwachs un enseignement de probabilités et statistiques à l'Institut Commercial Supérieur de Strasbourg 9. Cet enseignement est conçu comme devant permettre de «donner un idée de la statistique à ceux qui ne sont pas très avancés en mathématiques ». Un livre, issu de ce cours, paraît en 1924 sous le titre Le calcul des probabilités à la portée de tous (1924) et est primé par l'Académie des Sciences ${ }^{10}$.

Le troisième symbole est une intervention lors d'un colloque. Présent à la « semaine de synthèse » consacrée à «La statistique, ses applications et les problèmes qu'elles soulèvent» organisée début juin 1935 par le Centre International de Synthèse fondé et dirigé par Henri Berr, Halbwachs est le représentant de la sociologie durant les neuf demies journées de ce colloque : il y côtoie notamment, Michel Huber, directeur de la Statistique Générale de France, les mathématiciens Émile Borel et Georges Darmois (Sorbonne), les historiens André Piganiol (Sorbonne) et Edmond Esmonin (Grenoble), le juriste Henri Lévy-Bruhl, les physiciens Max Born (Cambridge) et Paul Langevin (Collège de France) ainsi que des représentants de la biologie et de l'histoire littéraire. Les actes seront publiés en 1944 par la Revue de synthèse. Dans sa contribution titrée «La statistique en sociologie», Halbwachs expose de façon synthétique, et pour la dernière fois de façon aussi précise, sa conception du rôle de la statistique dans l'étude des phénomènes sociaux. Cet article représente le point culminant de ses efforts pour expliciter les risques mais aussi le pouvoir des statistiques et des mathématiques.

Halbwachs n'a toutefois pas été qu'un simple utilisateur ou penseur de la statistique. Ses diverses responsabilités institutionnelles dans des instances « statistiques », notamment à partir des années trente, l'ont amené à participer à la production statistique ou en tout cas à la coordination de cette production ${ }^{11}$ : membre de la Société Statistique de Paris, membre de l'Institut International de Statistique depuis 1935, délégué français à la Conférence des statisticiens du travail en 1936 puis 1938. Et à diverses reprises, il cherche à donner une forme institutionnelle plus explicite à son intérêt pour la

\footnotetext{
${ }^{9}$ Index Generalis (Annuaire Général des Universités), 1923-1924, F153 et 1924-1925, F153.

${ }^{10}$ Ces informations sont extraites d'une lettre de Fréchet à Lazarsfeld en 1964 (GEMELLI, 1998, 491). Dans cette lettre, Fréchet affirme qu'Halbwachs ne faisait qu' « assister à ces cours » mais, comme l'attestent les volumes de l'Index Generalis des années 1923 à 1925, F149, Halbwachs et Fréchet étaient tous les deux en charge de ce cours. Par ailleurs, si Halbwachs côtoie le mathématicien Cerf, ce dernier n'a jamais collaboré à l'ouvrage de Fréchet et Halbwachs ; même si une lecture un peu rapide de l'article de CRAIG (1979) a pu le laisser croire à plusieurs auteurs, notamment BOURDIEU $(1987,165)$ et BAUDELOT, ESTABLET $(1994,11,47)$.

${ }^{11}$ ALEXANDRE, 1940-1948, 3.
} 
statistique sociale et ses usages. Ainsi, en 1930, apprenant que la Sorbonne a demandé la création d'une chaire de «sociologie statistique », il se place aussitôt dans le rang des postulants. Cette chaire ne sera pas créée mais il semble avoir eu la ferme intention d'occuper ce poste ${ }^{12}$. Autre exemple : à la mort de François Simiand, en 1935, Halbwachs qui apparaît comme son héritier naturel, envisage de présenter sa candidature au Collège de France. Marcel Mauss, titulaire d'une chaire depuis 1931, souhaite cette candidature car, selon lui, Halbwachs représente « les mêmes méthodes quantitatives dont la négligence en France est si criminelle ». Halbwachs renonce finalement à se présenter et préfère poursuivre une carrière à la Sorbonne: il espère justement y sauver «la tradition des méthodes quantitatives $»{ }^{13}$. Cet épisode d'une candidature au Collège de France nous renvoie six ans plus tôt lorsque, évoquant déjà une possibilité de candidature finalement retirée au profit de Mauss, Halbwachs s'interroge sur la possibilité de créer, selon ses propres mots, une « chaire de statistique morale $»{ }^{14}$.

Afin de dépasser cette image, évocatrice mais peu explicite quant au fond, d'un sociologue soucieux de statistique, il est essentiel de déterminer ce que cette image renferme exactement: quelle était sa conception des statistiques et de leurs rôles dans le raisonnement sociologique ? Plus précisément, il nous semble nécessaire d'éclairer les pouvoirs qu'Halbwachs attribue à la statistique, les limites qu'il lui assigne et les conditions d'utilisation qu'il estime nécessaire de respecter.

L'interrogation ne doit toutefois pas s'arrêter là : une question est de parvenir à saisir la conception de la statistique, de ses instruments et de ses usages chez Halbwachs ; une autre question est de chercher à comprendre dans quelle mesure cette conception, parfois énoncée de façon très générale, s'articule avec le reste de son œuvre, ou au moins avec les grandes lignes de forces de son œuvre. En particulier, il est indispensable de s'interroger sur les liens pouvant exister entre sa pensée sur les groupes/classes, sur les catégories sociales, et sa conception de la statistique. Bien que naturelle, cette question trouve peu de réponses dans la littérature consacrée à Halbwachs.

\section{I - La statistique au cour d'une sociologie positive}

L'énoncé est sans ambiguïté : pour Halbwachs, la recherche statistique est «le seul moyen de reconnaître les régularités sociales ${ }^{15}$. La généralité de

\footnotetext{
${ }^{12}$ CRAIG, 1979, 290.

${ }^{13}$ Lettres de Mauss : FOURNIER, 1994, 656-657.

${ }^{14}$ Lettre d'Halbwachs à Mauss, Strasbourg, 11 juin 1929 : FouRNIER, 1994, 568.

${ }^{15}$ HALBWACHS, 1944 (1935), 114.
} 
cet énoncé appelle des précisions. Elles sont de divers ordres. Débutons par l'examen des capacités qu'il attribue à la statistique.

Première capacité : la statistique est un instrument de connaissance permettant au sociologue d'accéder à ce qui n'est pas apparent. Les "procédés de représentation schématique, d'analyse et de simplification » qu'offre la statistique «procurent une connaissance plus claire des données dont nous disposons, et ils nous font découvrir entre elles bien des rapports que nous n'apercevions pas avant de les avoir ainsi élaborées » ${ }^{16}$.

Deuxièmement, Halbwachs estime que la statistique permet de remplacer l'expérimentation qui est impossible en sciences sociales. Soucieux, comme le montrent les récurrentes comparaisons qu'il établit entre les sciences sociales et les sciences physiques dans ses écrits, de justifier et de valoriser la méthodologie des sociologues, Halbwachs s'appuie très directement sur Statistique et expérience de Simiand (1922) pour défendre l'idée que la sociologie peut arriver à disposer d'outils lui permettant de remplacer la pratique expérimentale. De ce point de vue, le sociologue est semblable au physicien qui « sans faire d'expérience, se [borne] à noter, au fur et à mesure des changements naturels de la température et de la pression, les changements du volume par exemple d'un gaz enfermé dans un ballon de substance élastique, fabriqué d'ailleurs pour des fins non scientifiques, et que, plusieurs fois, l'observation eût été faite par hasard à une température identique ${ }^{17}$. Construisant l'un et l'autre un tableau récapitulant leurs résultats, le physicien obtient des données en tout point semblables à celles obtenues par le sociologue examinant le social. Au fond, expérimentation et opération statistique se rejoignent et il n'y a pas de différences fondamentales entre, par exemple, les données recueillies par Mendel dans ses études expérimentales sur les pois de jardins, et les données statistiques de Galton sur la transmission de la couleur des yeux ${ }^{18}:$ «les opérations statistiques présentent tous les caractères d'une méthode expérimentale ».

\section{II - La statistique pour saisir le collectif et son hétérogénéité}

Troisièmement, et c'est là que se situe la vertu principale de la statistique aux yeux d'Halbwachs, elle permet de saisir ce qui échappe à l'examen des seuls individus : les traits du collectif. Puisque les «tendances, croyances et pensées collectives sont représentées, inégalement, diversement, par chaque individu » et que «chacun n'en présente qu'une partie ou qu'un aspect», il est indispensable de «rassembler toutes ces parties, de les dénombrer

\footnotetext{
${ }^{16}$ Ibid., 123.

${ }^{17}$ HALBWACHS, 1923, 345

${ }^{18}$ Ibid., 347-352, 370.
} 
intégralement, de façon à ne négliger aucune d'entre elles, et d'en recomposer l'ensemble ${ }^{19}$. Ainsi, si la «statistique s'applique aux faits qu'on détermine quantitativement au moyen d'un nombre plus ou moins grand de constatations individuelles », elle s'applique en réalité « à des faits qui sont distincts de ces éléments individuels, et qui ne sont réalisés comme tels en aucun d'eux » : « la statistique permet d'atteindre les caractères d'un groupe, qui ont une réalité pour le groupe entier, mais qu'on ne découvriraient dans aucun membre de ce groupe pris à part et isolément ${ }^{20}$. Le meilleur exemple est certainement «la durée moyenne de vie dans un groupe d'hommes, qui ne sera, en général, la durée de vie exacte d'aucun de ces hommes pris au hasard ${ }^{21}$.

Penser le groupe et penser la régularité ne s'opposent toutefois pas, chez Halbwachs, à la recherche de la variabilité, c'est-à-dire à la fois aux différences entre individus et aux évolutions temporelles. Malgré son apparente simplicité, la formule « la recherche statistique est le seul moyen de reconnaitre les régularités sociales » ne doit en effet pas nous tromper. Dans l'esprit d'Halbwachs, régularité ne s'oppose pas à hétérogénéité. Un fait social peut prendre, dans une société et un temps donnés, des formes diverses. Or le calcul de moyennes peut faire disparaître cette diversité. Pour cette raison au moins, toute analyse ne doit pas se contenter de calculer des moyennes: «pour être assuré que [les] moyennes [correspondent à la réalité], il faut que le statisticien calcule des valeurs typiques complexes aussi, par exemple des médianes, avec des quartiles, des déciles et qu'il en calcule plusieurs de divers genres, qu'il multiplie les recoupements ${ }^{22}$. Il rejoint en cela une position également défendue par Simiand (1912): «Défions-nous des moyennes: contrôlons, recoupons les indications de moyennes d'un type et d'autres indices, par des données complémentaires ${ }^{23}$. C'est ce principe qu'il applique dans ses recherches sur les budgets ouvriers, traquant les différences derrière les moyennes: il parvient de cette façon à montrer que « dans une même catégorie de revenus, des ouvriers, des groupes d'ouvriers très différents se trouvent fondus $»{ }^{24}$.

Ainsi, à la différence de Durkheim qui soulignait et développait une théorie sociologique de la régularité, de la constance et de l'invariance, Halbwachs porte davantage son regard sur la variabilité, sur les écarts, sur l'élasticité. Il ne nie pas l'existence de régularités mais les estime inséparables des évolutions et des variations, des processus dynamiques.

\footnotetext{
${ }^{19}$ Halbwachs, 1944 (1935), 116.

${ }^{20}$ Ibid., 114 .

${ }^{21}$ Ibid.

${ }^{22}$ Ibid., 124.

${ }^{23}$ Halbwachs, 1923, 363 ; voir aussi HaLbWaChS, 1930, 4-5.

${ }^{24}$ HALBWACHS, 1912a, 332.
} 
D'autre part, comment la société pourrait rester immuable alors que des changements techniques interviennent régulièrement, alors que les modes de production et donc les métiers changent? Les modes de consommation ont, par exemple, changé depuis l'arrivée de l'électricité dans les foyers. Plus généralement il refuse l'idée d'équilibre, estimant que tout est dynamique, tout est en déséquilibre permanent : « il faut qu'il y ait un hiver pour qu'il y ait un printemps ${ }^{25}$. C'est cet argument qui lui permet de critiquer, de façon radicale, toute tentative cherchant à établir les lois de l'équilibre (économique notamment) dans les sociétés, et a fortiori toute tentative cherchant à « établir une vie économique en équilibre $\gg{ }^{26}$. Reprenant à nouveau à son compte des positions défendues par Simiand, il estime que la vie économique est un passage perpétuel d'un état de déséquilibre à un autre état de déséquilibre, et que cette succession d'état est justement la condition nécessaire au progrès économique et social.

\section{III - Les limites des statistiques}

Pourtant, aussi utiles et indispensables soient-elles, les analyses statistiques ne sont pas des fins en soi : elles ne dispensent en effet pas d'une analyse sociologique approfondie permettant de comprendre la logique sociale présidant aux résultats statistiques. Par exemple, l'analyse statistique des budgets des familles ouvrières doit être complétée d'une recherche des raisons sociales permettant d'expliquer les différences observées entre groupes et l'homogénéité relative des comportements au sein de certains groupes. Nous savons qu'il trouvera les raisons du côté des « représentations collectives ». L'essentiel étant ici pour nous qu'Halbwachs ne conçoit pas les statistiques comme des informations permettant au sociologue de se passer d'une recherche approfondie des causes mais, bien au contraire, comme de simples informations, de simples traces, de la réalité sociale ; informations et traces qui constituent la matière première de la réflexion sociologique. À ces yeux, les statistiques ne disent rien de la nature des phénomènes qu'elles saisissent : pour saisir cette nature, il faut dépasser ces données et se livrer à l'analyse sociologique. C'est ce qu'il exprime dans cet extrait de sa conclusion des Causes du suicide: « les statistiques nous indiquent combien il y a eu de suicides dans un groupe. Mais elle ne nous font pas connaître à quel ordre de faits sociaux, domestiques, religieux, politiques, économiques chaque catégorie d'entre eux se rattachent. Un ensemble de suicides est donc une donnée très complexe qu'on ne peut mettre en rapport qu'avec un

\footnotetext{
${ }^{25}$ HaLBWACHS, 1937c, 399.

${ }^{26}$ Ibid.
} 
ensemble complexe d'influences ${ }^{27}$. Il reprend cette idée sans rien en changer en 1938, dans Morphologie sociale: "Les statistiques en ellesmêmes ne peuvent rien apprendre sur la nature des faits mais seulement faire apparaître leurs variations notables ». En d'autres termes, peut-être de façon plus radicale, l'analyse statistique ne constitue pas une véritable analyse sociologique : le chercheur ne peut en aucune manière croire conduire un raisonnement sociologique en réalisant des analyses statistiques. «Les méthodes statistiques en sociologie quantitative [...] ne nous apportent pas des théories, mais des instruments d'observation et de comparaison, à la fois précis et objectifs » ${ }^{28}$. Ces méthodes viennent à l'appui de la recherche positive mais ne s'y substitue pas: Halbwachs refuse aux statistiques et aux mathématiques un pouvoir explicatif ou compréhensif. Elles ne constituent à ces yeux que des techniques, venant soutenir le sociologue: «En science sociale, c'est en adaptant la statistique mathématique aux problèmes que pose la recherche positive, en lui demandant surtout les moyens d'obtenir plus de précision dans nos mesures, et des moyens d'expression plus exacts, que nous aurons chance de progresser un peu ${ }^{29}$. Elles ne sauraient se substituer au travail d'analyse et d'interprétation du sociologue puisque, «quant aux explications, il y a tout lieu de penser qu'elles ne sauraient être du type mathématique $»^{30}$. Le rôle assigné par Halbwachs à la statistique est donc modeste ; même s'il est réel et indispensable.

En somme, les statistiques, la statistique, ne parlent pas d'elles-mêmes : en tout cas elles ne parlent pas «correctement» d'elles-mêmes. Le sens sociologique des résultats statistiques ne vient pas spontanément, même si les statistiques véhiculent du sens. Considérant par exemple l'usage de la moyenne dans la théorie de l'homme moyen de Quetelet (sur laquelle nous reviendrons plus loin), Halbwachs estime que Quetelet élabore «une conception générale intéressante et profonde des faits sociaux ${ }^{31}$ et que cette théorie est liée à sa conception de la moyenne. L'usage des outils statistiques n'est jamais neutre et dénué de tout a priori théorique. Il est donc nécessaire d'identifier scrupuleusement les sens et limites des indicateurs statistiques. C'est par exemple ce qu'il fait en 1933, lorsqu'il s'interroge sur la portée de l'indice du coût de la vie : «il ne faut demander à une expression statistique que ce qu'elle peut nous donner, que ce que veulent atteindre ceux qui la calculent. L'indice du coût de la vie nous apprend quelle influence exercent, sur un genre de vie supposé identique, les variations des prix de détail. Il ne prétend point nous révéler les variations de genre de vie lui-même [...]. Il se

\footnotetext{
${ }^{27}$ HALBWACHS, 1930, 492.

${ }^{28}$ HalBwaChS, 1944 (1935), 127-128.

${ }^{29}$ Ibid., 134 .

${ }^{30}$ Ibid.

${ }^{31}$ HaLbwachs, 1912b, 2.
} 
peut que, d'une période à l'autre, la répartition des dépenses change plus qu'on ne croit. L'indice du prix de la vie ne prétend pas nous apprendre comment varient les besoins, puisqu'il suppose qu'ils ne varient pas ${ }^{32}$. La formalisation mathématique, les formules statistiques, ne sont jamais dénuées de sens mais ce sens doit être attentivement identifié et les formules doivent être utilisées en ayant ce sens présent à l'esprit.

On ne peut d'ailleurs pas utiliser aveuglement les instruments statistiques sous peine de créer des artifices ou des indicateurs dont le sens sociologique nous échapperait. Notamment, toute application d'une formule ne fournit pas une «statistique pertinente» c'est-à-dire sociologiquement significative : «tout comptage n'est pas une statistique ${ }^{33}$. De la même manière, tout ensemble ne constitue pas un groupe social : «il n'y a d'ensembles réels que les groupes sociaux, précisément parce qu'ils sont constitués par des éléments différents. Tous les autres ensembles sont des collections. Les espèces humaines sont des collections d'organismes, les organes et tissus sont des collections de cellules, et l'organisme lui-même n'est qu'un individu. Les groupes sociaux sont plus encore, et autre chose ${ }^{34}$. Se situant dans le droit fil de la pensée de Simiand, Halbwachs estime qu' ' il ne suffit pas de compter un grand nombre d'unités ou de cas pour obtenir une statistique. Un calcul de ce genre n'offre en effet d'intérêt pour le savant que s'il s'applique à quelque ensemble, à quelque groupe ayant une certaine consistance, ou soupçonné d'avoir une certaine consistance, en tant qu'ensemble, en tant que groupe». Il reprend à son compte les fameux exemples de Simiand: "le kilométrage d'une station de chemin de fer à toutes les stations du réseau n'est pas un fait statistique ; le nombre de fois où un certain jour de la semaine se rencontre au cours d'un mois n'est pas un fait statistique $»^{35}$.

Arrêtons-nous sur ces dernières citations d'Halbwachs car elles permettent se saisir une des conditions essentielles posées à l'utilisation des statistiques. Quelle est la condition primordiale pour qu'une statistique ait un sens? Si la saisie des caractéristiques collectives repose sur le calcul de statistiques, inversement, l'existence du collectif est une condition indispensable à l'usage de statistiques : «il faut que ces groupes présentent une certaine réalité, une certaine consistance, en tant que groupes ${ }^{36}$. Toute série de fait ne constitue pas un phénomène social : «la moyenne de prix recueillis à dix ans, ou même à cinq ans de distance, n'est pas une statistique, tant qu'on ne sait pas s'il y a une liaison ou un rapport de ressemblance entre

\footnotetext{
${ }^{32}$ HALBWACHS, 1933, 2.

${ }^{33}$ HALBWACHS, 1944 (1935), 115.

${ }^{34}$ Ibid., 116.

${ }^{35}$ HALBWACHS, 1923, 353.

${ }^{36}$ HALBWACHS, 1944 (1935), 114.
} 
ces années au point de vue économique, et quels mouvements se sont produits de l'une à l'autre ${ }^{37}$. Et toute collection d'individus ne constitue pas une classe ou un groupe social : une classe existe si «elle prend conscience d'elle-même ». Il affirme à diverses reprises que si «rien n'empêche un historien ou un sociologue de distinguer dans une société beaucoup de groupes, en tenant compte des ressemblances et des différences extérieures ou apparentes entre leurs membres », «de tels 'arrangements' ont chance d'être le plus souvent artificiels ${ }^{38}$. Un groupe défini par un simple critère statistique ou démographique peut notamment présenter un caractère artificiel : «si notre âge est en quelque sorte imposé par la société, il ne s'ensuit pas que les hommes ou les femmes d'un âge donné [...] constituent un groupe social défini. [...] Bien qu'on ait proposé de former, par exemple, le parti des hommes de 40 ans, s'il y a des intérêts et préoccupations communs aux jeunes gens, aux adultes, aux gens plus âgés, il s'agit en réalité de groupes dont les membres ont des âges assez divers, et qui s'espacent sur un plus large intervalle ${ }^{39}$.

En cela, Halbwachs se situe dans le droit fil d'une tradition qui, à travers Quetelet puis Adolphe Bertillon, estime que seule la consistance des groupes peut justifier le calcul statistique. Mais Quetelet puis Bertillon défendaient l'idée que la forme normale d'une distribution de valeur est un indice de la réalité, de l'existence, d'un groupe ${ }^{40}$. Halbwachs n'utilise pas ce principe, qu'il rejette fermement. Pour lui, l'existence sociale d'un groupe, le fait qu'un ensemble d'individus constitue une classe, proviennent de l'existence d'une conscience collective au sein d'un groupe: «appeler classe un ensemble d'hommes dans lequel une conscience de classe ne s'est point développée et ne se manifeste pas, c'est ne désigner aucun objet social, ou c'est désigner une classe en voie de formation qui n'existe pas encore ${ }^{41}$.

Sa définition des classes sociales, à la fois du point de vue théorique et d'un point de vue pratique ne repose jamais sur des critères statistiques ou quantitatifs. Au regard de l'analyse statistique qu'il conduit, sa définition des groupes ou des classes est une définition a priori. Elle n'est justifiée par aucun critère mathématique mais seulement par des arguments sociologiques. C'est notamment le cas en 1912, dans les premières pages de sa thèse de doctorat où il soulève la question des "limites et de l'unité de la classe ouvrière ${ }^{42}$ : c'est à partir de l'étude «qualitative» des modes de vie, des traditions, des intérêts, des consciences (notamment par rapport aux autres

\footnotetext{
${ }^{37}$ Ibid., 115.

${ }^{38}$ HALBWACHS, 1912a, II.

${ }^{39}$ HalbwaCHS, 1944 (1935), 118-119.

${ }^{40}$ DESROSIÈRES, 1991, 251 sqq.

${ }^{41}$ HALBWACHS, 1912a, II ; c'est Halbwachs qui souligne.

${ }^{42}$ Ibid., 1-124 (livre I).
} 
groupes sociaux), des compétences professionnelles, des techniques utilisées (outils, instruments, machinisme...) qu'il définit la classe ouvrière (et assimile à celle-ci les groupes paysans). Son usage de l'enregistrement et de l'analyse statistique est exclusivement dédié à l'étude des «besoins et dépenses des habitudes consommatrices des ouvriers ${ }^{43}$, jamais à la définition et l'identification d'une classe.

Ce constat est confirmé par un examen attentif des critères permettant à Halbwachs de définir les classes sociales. Il est possible d'en identifier quatre mais aucun ne fait référence à des analyses ou des arguments statistiques : ni la définition par les modes, styles et niveaux de vie; ni la définition par le contenu des actes de travail ; ni la définition par les consciences individuelles et collectives ; ni la définition par la mémoire collective ${ }^{44}$.

Le cours qu'il professe à la fin des années trente à la Sorbonne sur Les classes sociales fournit un autre témoignage de cet état de fait : il n'y est jamais question de définition statistique ou de critères quantitatifs pour définir les classes. Et si, en conclusion de ce cours, il avoue estimer difficile de définir précisément les classes, la possibilité d'une définition statistique ne semble pas lui venir à l'esprit : « La classe est un groupement sui generis qui se présente sous des formes assez mal définies, assez estompées. [...] Pourtant nous sentons qu'il y a là une réalité sociale bien effective ${ }^{45}$.

En somme, sa conception des statistiques leur attribue des capacités relativement modestes qui doivent être contrôlées par le sociologue. Celui-ci ne saurait confier l'analyse de ses données statistiques à la seule logique formelle et à la seule loi des mathématiques.

\section{IV - Contre les abstractions mathématique et graphique}

En effet, dans son travail d'élaboration d'une connaissance positive, le sociologue a recours à deux logiques, de deux ordres de faits et d'arguments : la logique ou l'ordre des faits empiriques; la logique ou l'ordre des instruments mathématiques qu'il utilise. Halbwachs hiérarchise très nettement ces deux logiques : le sociologue doit suivre la logique des faits empiriques, quitte à s'aider, pour leur analyse, de ces instruments mathématiques. Pour illustrer les dangers qu'il y a à se laisser absorber par la seule logique mathématique, il propose une métaphore médicale: "Que dirions-nous cependant d'un médecin, appelé chez un malade qui souffre peut-être de troubles nerveux depuis longtemps, neurasthénie, ou cyclothymique, et qui s'en tiendrait à utiliser les instruments perfectionnés

\footnotetext{
${ }^{43}$ Ibid., 136.

${ }^{44}$ MONLIBERT, 1997.

${ }^{45}$ HALBWACHS, 1937a, 174.
} 
qu'il a apportés, thermomètre, appareils pour mesurer la pression, pour observer par radioscopie l'intérieur du corps ? Ne serait-il pas essentiel qu'au contraire il prenne d'abord, en l'interrogeant, une vue d'ensemble de l'évolution suivie par la maladie jusqu'à ce moment : et n'est-ce pas grâce à ce qu'il en apprendra que les observations précises, qu'il peut faire avec ses instruments, recevront tout leur sens ? $\gg{ }^{46}$.

C'est la logique du social, de l'empirie, qui doit primer sur la logique des mathématiques, la logique des statistiques ou, selon une formulation plus contemporaine, la logique de la logique. Il ne faut pas s'imaginer «qu'on a expliqué la réalité quand on lui a substitué une formule ou une figure avec laquelle elle cadre à peu près. On risque alors de superposer aux groupes réels des groupes fictifs qui ne paraissent pas correspondre aux premiers que parce qu'ils ne sont que ces premiers en effet, mais privés d'une grande partie de leur contenu $\gg{ }^{47}$. Il ne faut pas que le recours à des expressions ou des figures mathématiques abstraites dispense le sociologue d'une analyse approfondie, systématique et fouillée, des faits sociaux qu'il étudie. Celui-ci doit procéder à de «multiples recoupements », doit ne pas hésiter à recourir à des analyses nombreuses qui se complètent les unes et les autres. Par exemple, les courbes «doivent suivre tous les replis du phénomène, le représenter dans toutes ses phases, mais aussi l'embrasser dans toute son étendue, et en toutes ses parties: c'est ainsi qu'on représentera les mouvements du salaire par plusieurs courbes, continues autant que possible, juxtaposées, aussi nombreuses qu'il y a de données correspondant à des groupes différents, agriculture, industrie, et diverses espèces et formes d'industrie, grandes villes, villes moyennes, petites villes. Cela exige un effort d'attention multiple, à la fois abstraite et concrète. Mais cette méthode empirique est la seule qui permette de rester en contact aussi étroit que possible avec la réalité ${ }^{48}$.

De cette remarque découle une nécessité : il est pour lui essentiel de traquer les biais de tous les instruments statistiques et mathématiques utilisés, en particulier, tous les aspects de la réalité sociale dont les instruments statistiques et mathématiques sont incapables de rendre compte. Par exemple, le coefficient de corrélation ne tient pas compte de l'enchaînement temporel des faits qu'il mesure : «L'inconvénient principal [de ce coefficient est de faire] abstraction de l'ordre dans lequel les variations se succèdent dans le temps, qui est pourtant loin d'être négligeable. On pourrait déplacer en même temps deux variations concomitantes de ce genre, les reporter du milieu au début, à la fin, ou, encore, prendre l'ordre de succession inverse, commencer par la fin: le coefficient de covariation serait le même: pourtant

\footnotetext{
${ }^{46}$ HALBWACHS, 1944 (1935), 133-134.

${ }^{47}$ Ibid., 123.

${ }^{48}$ Ibid., 124.
} 
l'enchaînement des faits serait tout autre car nous sommes dans un domaine où les successions de phénomènes ne sont pas réversibles ${ }^{49}$. Il ne s'agit pas de nier tout intérêt à la méthode des variations concomitantes, mais il faut «comprendre qu'une variation peut avoir une signification très différente, bien qu'elle reste la même quant au sens et à l'intensité, suivant la phase de l'évolution où elle se produit ». Dans certains cas, cette méthode et cet indicateur statistique peuvent laisser échapper l'essentiel ${ }^{50}$.

Il y a donc, aux yeux d'Halbwachs, un véritable danger à simplifier à l'excès en ramenant la réalité sociale à une formalisation trop pure. La recherche de propriétés mathématiques simples portant sur des données ne saisissant qu'une infime partie de la réalité est une démarche vaine, artificielle et dangereuse : vaine puisqu'elle oublie un grand pan de la réalité sociale ; artificielle car elle produit des représentations et des résultats dont la logique est mathématique et non sociologique; dangereuse car elle laisse croire que l'identification de propriétés mathématiques constitue une fin en soi. Même les pyramides des âges constituent un exemple de ce qu'Halbwachs conçoit comme étant un artifice mathématique, comme une représentation dénuée de véritable sens sociologique : «il y a beaucoup trop de rigueur arithmétique, une rigueur un peu artificielle et arbitraire, dans les présentations $[\ldots]$ de pyramides des âges. [...] C'est bien là une traduction exacte des données numériques. Mais quelle en est la portée, au point de vue sociologique? $\gg^{51}$. Comparer deux pyramides pour deux groupes sociaux différents ou deux pays, en ne tenant pas compte des différences sociales pouvant exister entre deux pays, notamment des différences concernant les frontières sociales entre la jeunesse et l'âge adulte, des différences de précocité dans le développement des individus, des différences dans la composition sociale des populations, est une démarche vaine et sociologiquement insignifiante. Sa critique est radicale: de la prise en compte des réalités sociales, « les pyramides des âges nous donnent une idée aussi schématique et aussi pauvre que les pyramides d'Égypte des destinées des multitudes humaines qui ont eu la tâche de les construire ${ }^{52}$. L'analyse des faits paraissant les moins sociaux, comme les naissances et les décès, ne peut pas échapper à la prise en compte du contexte social, de leurs dimensions sociales : " la mort est un fait biologique, mais c'est aussi un fait social. Elle peut varier, le taux de mortalité peut varier, non seulement pour la population tout entière, mais pour chaque catégorie d'âge, précisément, suivant la composition par âges de la population (qui résulte de certaines conditions sociales) [...] La mort résulte de la vie, et la vie est conditionnée

\footnotetext{
${ }^{49}$ Ibid., 129-130 ; voir aussi HALBWACHS, 1937 b.

${ }^{50}$ Ibid., 130.

${ }^{51}$ Ibid., 119-120.

${ }^{52}$ Ibid., 120.
} 
par la situation économique, par l'organisation de la famille, par les institutions et par les coutumes ${ }^{53}$. Sa condamnation de l'abstraction est donc sans appel: «De même que l'homo oeconomicus, un homo demographicus est une abstraction trop soigneusement détachée de la réalité pour nous apprendre quoi que ce soit sur le réel $\gg{ }^{54}$.

Et puis, faire trop de mathématiques, faire appel à trop de formalisation pour saisir le social, c'est prendre le risque de trop se désintéresser des réalités positives. C'est oublier les faits de la réalité sociale ; c'est se laisser être « trop occupé par [des] équations et [des] formules pour interroger les faits eux-mêmes en leur complexité mieux saisie ${ }^{55}$.

\section{V - Contre les excès de la standardisation}

Ce refus de la simplification rejoint un autre refus d'Halbwachs : celui de la standardisation excessive des situations à des fins de comparaison statistique. Ces deux travers sont liés par une raison logique simple: non seulement la mise en équations ou en courbes mathématiquement définies est une démarche qui cache l'essentiel, mais elle peut aussi faire disparaître cet essentiel, le faire disparaître derrière une régularité forcée ou une simplification excessive. «On peut craindre que les simplifications nécessaires pour l'établissement de ces courbes aient éliminé un grand nombre de particularités essentielles qui seules nous mettraient sur la voie de l'explication ${ }^{56}$. En particulier, la création de populations fictives par des procédés statistiques n'a pas de sens sociologique : «tout se passe comme si, pour étudier les caractères démographiques d'un pays, il fallait partir d'une population qui n'est celle d'aucun pays, comme si l'on avait à faire à des hommes qui ne naissent, ne se marient, ne meurent dans aucune région définie de quelque manière, quant aux coutumes familiales, religieuses, juridiques, économiques $\gg{ }^{57}$. Ce type de raisonnement conduit à des situations ou à des «problèmes bien paradoxaux»: «Combien de temps vivraient les Français si, restant Français, ils vivaient dans les mêmes conditions physiques et sociales que les Suédois? Combien de temps vivraient les Allemands si, restant Allemands, ils vivaient dans les mêmes conditions que les Français ?». Cela revient, comme l'observait « plaisamment » avant lui Simiand à propos des comparaisons des niveaux de vie entre plusieurs pays, à « calculer ce que coûterait en Laponie un éléphant

\footnotetext{
${ }^{53}$ Ibid., 121-122.

${ }^{54}$ Ibid., 123.

${ }^{55}$ HALBWACHS, 1939, 313.

${ }^{56}$ HALBWACHS, 1944 (1935), 125.

${ }^{57}$ Ibid., 122-123.
} 
de l'Inde, s'il vivait en Laponie comme un éléphant vit dans l'Inde, ou ce que coûterait dans l'Inde un renne, s'il vivait dans l'Inde comme un renne vit en Laponie ${ }^{58}$. Question cocasse et sans véritable intérêt que se demander, autre exemple, «comment vivrait un chameau, si, restant chameau, il était transporté dans les régions polaires, et comment vivrait un renne si, restant renne, il était transporté dans le Sahara ${ }^{59}$.

Pour les mêmes raisons, Halbwachs s'oppose aussi à l'étude de statistiques concernant des groupes ou des ensembles de trop grande taille, qui nécessairement regroupent des réalités très diverses. Saisir les faits sociaux à trop grande échelle, c'est créer une standardisation au moyen d'instruments mathématiques ou statistiques ; standardisation qui n'a pas de consistance sociologique.

\section{VI - Contre les excès de la décomposition analytique}

Son refus de voir construire des populations fictives permettant de raisonner «toutes choses égales par ailleurs » le conduit à concevoir comme impossible l'identification des effets séparés des facteurs sociaux: «Lorsqu'on compare deux régions, l'une à majorité catholique, l'autre à majorité protestante, il ne faut pas oublier qu'elles diffèrent sous d'autres rapports qu'au point de vue religieux. Tantôt les catholiques et les protestants se rattachent à des nationalités différentes [...]. Ailleurs, les catholiques sont des paysans, les protestants vivent dans les villes, ou dans des régions très urbanisées et se trouvent engagés dans les professions industrielles et commerciales. La religion n'est pas sans jouer un rôle, mais, sans doute, un rôle assez restreint. En tout cas, il est impossible d'étudier séparément l'influence de la religion et l'action des autres facteurs ${ }^{60}$. Et s'il corrige, comme nous le savons, le travail de Durkheim sur le point essentiel du rôle de la religion dans les suicides, ce n'est peut-être pas tant pour rectifier une erreur factuelle, que pour montrer que la conclusion à laquelle aboutissait Durkheim en 1897 était plus ambiguë, plus ténue, que celui-ci ne voulait le croire. «Que les diverses confessions religieuses produisent, comme telles, plus ou moins de suicides, c'est une des conclusions de l'étude entreprise par Durkheim qui impressionnent le plus mais c'est peut-être aussi la plus discutable ${ }^{61}$. En effet, selon lui, « un ensemble de suicides est une donnée très complexe qu'on ne peut mettre en rapport qu'avec un ensemble

58 Simiand, 1930, Cours d'économie politique, Paris, Domant-Montchrestien, 288 ; cité dans HALBWACHS, 1933, 8.

${ }^{59}$ HALBWACHS, 1944 (1935), 122.

${ }^{60}$ HaLBWACHS, 1930, 491-492.

${ }^{61}$ Ibid., 492. 
complexe de causes. C'est ce qu'on l'on tend à appeler aujourd'hui un fait de sociologie totale, qui s'explique non point simplement par un facteur, mais par un système d'influences. Ces ensembles complexes de facteurs et de circonstances, ce peuvent être des régions, des régions définies non pas du point de vue géographique mais comme des zones de civilisation ${ }^{62}$.

Ainsi, s'il s'oppose fondamentalement à toute idée ou toute démarche trop abstraite, c'est-à-dire à toute démarche cherchant à extraire de la réalité sociale un petit nombre de faits parfaitement circonscrits et à les détacher de leur contexte, c'est parce qu'il estime que les processus d'abstraction ou de décomposition analytique des causes est artificiel et établit des frontières entre des faits interdépendants. Si c'est un «progrès, en démographie, d'avoir mis en lumière l'influence de la mortalité et de la natalité sur la répartition des âges », cette représentation des phénomènes est «trop abstraite » : « toute population doit être replacée dans un milieu à la fois social et matériel [...] Il faut tenir compte des forces qui tendent à accroître ou à maintenir à son niveau la natalité et de celles qui lui font obstacle [...] Le sexe, l'âge, la mortalité, la natalité ne sont pas des faits simplement vitaux ». Il lui paraît indispensable « d'embrasser dans son ensemble et dans ses rapports avec les autres » tout « l'ordre des réactions collectives ${ }^{63}$.

$\mathrm{Si}$ une décomposition des causes et des facteurs est impossible en sciences sociales, les sciences physiques, qui peuvent pourtant élaborer des expériences, ne sont pas mieux loties: «si on réussissait à isoler matériellement chacun des composants d'un groupe d'ensemble, on étudierait l'action de chacun des facteurs sans être gêné par l'action des autres. Mais y parvient-on, même dans les sciences physiques? [Prenons un exemple] : voici un ensemble d'observations sur les variations de volume d'une même quantité de gaz. Puis-je isoler l'action de la pression, puis l'action de la température, et les étudier séparément ? Oui, par un artifice, c'est-à-dire en fixant dans le premier cas la température, dans le second la pression ; mais si je les fixe, je ne les supprime point $»^{64}$.

En fin de compte, s'il s'oppose si fermement à toute démarche de standardisation ou de décomposition analytique excessives, c'est parce qu'il conçoit la sociologie comme une activité devant étudier les différences. «En réalité, écrit-il, c'est la différence qui nous intéresse » : «si on élimine ou neutralise toutes les différences [...] on peut se demander ce qu'il reste, et si, d'élimination en élimination, on n'en est pas arrivé à se retirer toute matière d'observation ${ }^{65}$.

\footnotetext{
${ }^{62} \mathrm{Ibid}$.

${ }^{63}$ HALBWACHS, 1938, 141-142.

${ }^{64}$ HalBWACHS, 1923, 369.

${ }^{65}$ Ibid., 365.
} 


\section{VII - Le meilleur contre-exemple : l'économie mathématique}

$\mathrm{Si}$ sa critique de l'abstraction mathématique est universelle et vise l'ensemble des sciences sociales, elle s'applique tout particulièrement aux économistes, qui voient fortement se développer les modèles mathématiques durant l'entre-deux-guerres (développement de l'économétrie ${ }^{66}$ ). Citant l'économiste et statisticien français Robert Gibrat qui affirme avoir ramené toutes les «courbes de répartition économiques à une courbe célèbre, la courbe en cloche dite aussi de Gauss ou des erreurs », Halbwachs enchaîne en s'interrogeant sur l'intérêt d'une telle démarche : «Est-ce bien vraiment l'idéal de la recherche statistique que de ramener les faits économiques et sociaux, leurs mouvements et leurs variations, à telle ou telle courbe avec laquelle les mathématiciens sont familiers ? ». "L'ingéniosité des algébristes et géomètres » est peut-être grande mais les «courbes de ce genre ne nous apprennent rien sur le mécanisme interne des liaisons entre diverses séries de faits d'où résultent les mouvements dont elles nous donnent une représentation approchée ${ }^{67}$. Plus généralement, il critique les revues économiques faisant une part trop belle à la statistique mathématique, et oubliant, par ce fait, les faits réels: «Je lis toujours avec beaucoup de considération pour les savants qui en sont les auteurs, les articles de statistique mathématique qui paraissent dans des revues telles que Metron et Econometrica. Dois-je avouer que je suis toujours un peu étonné d'y voir si rarement affleurer la réalité positive, et de l'indifférence qu'on paraît y témoigner d'ordinaire aux faits purs et simples, aux faits dans leur ampleur et leur détail, et dans leur extrême variété ? " ${ }^{68}$. Si la science économique manque parfois, selon lui, de précision et d'exactitude, et se contente d'accumuler une érudition un peu vaine, l'excès de mathématique et de formalisation abstraite est une démarche tout aussi vaine et constitue un danger tout aussi grand pour la recherche du vrai savoir. Son jugement est sévère et sans appel : il estime que "l'économie mathématique est surtout intéressante à titre d'application des mathématiques et pour les mathématiciens $"{ }^{69}$. Pour les autres, l'économie mathématique n'est qu'un «beau roman » (sic) puisqu'elle ne renseigne pas véritablement sur la réalité sociale, sur les faits humains et sociaux : «j'ai lu Cournot, j' ai lu Walras, j'ai lu Pareto, et je dois dire qu'ils ne m'ont pas appris grand-chose sur la réalité même ${ }^{70}$. Au fond, la mathématisation ne fait pas qu'une science devienne positive, réelle.

\footnotetext{
${ }^{66}$ Armatte, 1995, 834-890 ; DesrosiÈres, 1993, 342-394 ; Le GALL, 1994.

${ }^{67}$ HaLBWACHS, 1944 (1935), 124-125.

${ }^{68}$ Ibid., 132-133.

${ }^{69}$ HaLbWaChS, 1937c, 406-407.

${ }^{70}$ Ibid., 407.
} 


\section{VIII - L'analyse halbwachsienne de la moyenne quetelesienne}

Sa pensée et son analyse de la démarche statistique en sociologie prennent un tour très concret dès qu'elles s'attachent à l'examen d'un instrument statistique particulier, la moyenne, et dès qu'elles visent une utilisation précise de cet instrument: l'utilisation qu'en fait Adolphe Quetelet pour élaborer sa théorie de l'homme moyen.

Bien que la thèse complémentaire d'Halbwachs ${ }^{71}$, présentant une étude de la théorie de l'homme moyen d'Adolphe Quetelet (1796-1874), soit un de ses tous premiers textes consacrés à l'analyse des instruments statistiques, cette thèse rassemble toutes les critiques développées plus longuement et plus théoriquement dans ses articles des années vingt et trente.

Un bref rappel de la théorie quetelesienne de l'homme moyen est ici opportun. Étudiant les distributions des traits physiques (taille, poids, crâne, mesures anthropométriques..) et moraux (mariage, décès, naissance...) de l'homme, constatant que ces distributions se repartissent de façon régulière et symétrique par rapport à la moyenne en suivant la courbe «normale » ${ }^{72} \mathrm{de}$ distribution des erreurs (courbe en cloche), Adolphe Quetelet en déduit l'existence d'un type humain idéal : tous les hommes sont des réalisations, des «copies », imparfaites de cet «homme idéal», qui possède toutes les caractéristiques moyennes. Cette déduction est le résultat de l'application (le « transport ») d'un résultat issu des sciences physiques (l'astronomie en particulier, qui est la première discipline de Quetelet) aux sciences morales et humaines : les mesures d'une grandeur unique (la position d'un astre, la taille d'un objet unique) se distribuent selon une courbe en cloche et la vraie mesure de cette grandeur est la moyenne de toutes les mesures (le sommet de la cloche).

S'il critique certains aspects fondamentaux de la théorie de l'homme moyen, Halbwachs ne remet toutefois pas en cause les capacités de Quetelet à être un «observateur scrupuleux des faits». Il ne l'accuse pas de mécanisme mathématique : Quetelet «a fait usage des mathématiques moins pour reconstruire hypothétiquement le réel que pour le mesurer avec précision et en exprimer les formes à l'aide de formules ou de courbes adéquates $\gg{ }^{73}$. Il ne l'accuse pas plus de vouloir proposer une théorie générale de la société : «Ce serait se méprendre sur le véritable caractère de son œuvre que d'y voir une de ces reconstructions idéologiques de la société où se complaisent tant d'auteurs, en particulier de philosophes et d'économistes ${ }^{74}$.

\footnotetext{
${ }^{71}$ HaLBWACHS, $1912 \mathrm{~b}$.

${ }^{72}$ Ce terme n'interviendra que plus tard, sous la plume de Pearson en 1894.

${ }^{73}$ HALBWACHS, 1912b, 4.

${ }^{74}$ Ibid., 5.
} 
Sa critique et son rejet de la théorie quetelesienne d'homme moyen n'en sont pas moins sévères et radicaux : «la théorie de Quetelet, qu'on l'applique aux faits biologiques [taille, poids], aux faits de population [naissances, morts], aux faits moraux [suicides, crimes, mariages...] n'est actuellement pas soutenable $»^{75}$. La propre sociologie d'Halbwachs, celle qu'il entend promouvoir, «s'inspire de principes opposés aux [principes quetelesiens] », même si les outils mathématiques sont identiques.

Ce qu'il reproche à Quetelet? Fondamentalement son interprétation et son usage de l'idée de moyenne. Son erreur est d'avoir assimilé une «moyenne arithmétique », qui exprime non pas une réalité mais le résultat d'un calcul, à une «moyenne véritable», calculée sur plusieurs mesures d'une grandeur qui existe réellement : « En prenant une moyenne, on peut en effet avoir en vue deux objets bien différents. On peut chercher à déterminer "un nombre qui existe véritablement" ou bien calculer un nombre qui donne l'idée la plus approchée de plusieurs quantités différentes, homogènes, mais de grandeur variable. On se trouve dans le premier cas, lorsqu'on mesure la hauteur d'un édifice vingt fois de suite: c'est là calculer une véritable moyenne ? On est dans le second cas, quand on calcule la hauteur moyenne des maisons qui se trouvent dans une rue déterminée : c'est une moyenne arithmétique ${ }^{76}$. Halbwachs estime inassimilables ces deux types de moyennes : l'une constituant un indicateur permettant de qualifier un groupe ou un ensemble d'objets ou de faits; l'autre renvoyant à la mesure d'une grandeur existant réellement. La ressemblance formelle de ces deux moyennes ne signifie par qu'elles sont identiques.

En somme, en assimilant une moyenne arithmétique à une moyenne véritable, Quetelet a cru établir que tous les phénomènes humains et moraux qu'il étudiait relevaient de la même logique que la mesure de grandeurs physiques uniques. Il a donc cru qu'il y avait «dans la nature des types déterminés et immuables " ${ }^{77}$ à partir desquels tous les phénomènes humains et naturels se déduisent. Au dehors des petites causes aléatoires qui sont, somme toute, relativement marginales, il existerait des causes uniques gouvernant la société et l'homme : un individu ne serait qu'une incarnation, approximative mais proche, d'un modèle unique d'homme ; les faits moraux ne seraient que des réalisations, approchées, d'un modèle unique. Et ces modèles, ces types, seraient identifiables par le seul calcul de la moyenne. Ce que Quetelet exprime en ces termes: l'«homme moyen» est «dans la

\footnotetext{
${ }^{75}$ Ibid., 10

${ }^{76}$ Ibid., 36-37.

${ }^{77}$ Ibid., 35.
} 
société l'analogue du centre de gravité dans les corps. Il est la moyenne autour de laquelle oscillent les éléments sociaux ${ }^{78}$.

Si Halbwachs rejette cette explication des faits humains et sociaux, il n'estime pour autant pas que le travail de Quetelet a été vain : il a en fait préparé les esprits à l'idée de l'existence de régularités sociales, de faits sociaux sui generis : " avec lui, sans doute pour la première fois, tout un groupe de savants, préoccupés d'observer les faits sociaux, ont eu le sentiment profond que ces faits, comme les autres, sont soumis à des lois rigoureuses ${ }^{79}$. Il a donc favorisé, d'une certaine manière, le développement d'une science de la société : la sociologie. «Peu importe la forme sous laquelle la science sociale s'est d'abord définie; l'essentiel était qu'elle se définisse, que la conviction s'ancrât, dans les esprits, qu'il existe en ce domaine, comme dans les domaines des autres sciences, un grand nombre de relations nécessaires. Cette conviction a été d'autant plus forte qu'on a pu croire que ces relations étaient, au fond, d'essence mathématique». Halbwachs reconnaît humblement que, si la sociologie positive bâtie depuis Durkheim conduit à des conclusions distinctes de celles de Quetelet, «il ne faut pas oublier que cette recherche elle-même n'a pu être poursuivie comme elle l'a été qu'à la faveur d'un état de l'opinion dont la sociologie est redevable en une large mesure à Quetelet et à ses théories » ${ }^{80}$. Quetelet est, pour Halbwachs, un maillon essentiel de l'histoire du développement d'une science positive des faits sociaux.

\section{IX - Le rejet de la théorie des erreurs en sociologie}

Par son analyse du statut des moyennes, Halbwachs montre que la similitude entre la situation du sociologue et celle du physicien, qu'il souligne pourtant dès qu'il le peut, n'est pas absolue. Derrière la question des moyennes se cache un point fondamental sur lequel le sociologue se distingue nettement du physicien : c'est le statut des «causes». Et cette différence renvoit à une autre différence profonde : le statut des probabilités dans les deux types de disciplines. C'est sur ce point que sa critique à l'encontre de Quetelet est la plus sévère.

Pour Halbwachs, l'erreur de Quetelet réside dans son recours injustifié de la théorie des probabilités : il construit sa théorie de l'homme moyen en faisant un parallèle entre les distributions obtenues à partir d'expériences aléatoires et les distributions des caractères physiques des hommes (taille,

\footnotetext{
${ }^{78}$ QUETELET, 1831, Recherches sur la loi de la croissance de l'homme; cité dans HALBWACHS, 1912b, 36 .

${ }^{79}$ HaLbWACHS, 1912b, 11.

${ }^{80} \mathrm{Ibid}$.
} 
poids...) ou des objets astraux (positions des astres). Il décrit ce parallélisme ainsi : " dans un jeu de hasard, sur un grand nombre de parties, les tirages se répartissent suivant leurs "chances" respectives, et permettent donc de reconnaître celles-ci : à côté de la probabilité $a$ priori, il y a une probabilité $a$ posteriori. Mais une série de mesures d'une même grandeur se répartissent autour d'une moyenne très régulièrement, et conformément à la loi des probabilités, comme si leur inexactitude résultait chaque fois d'une combinaison de causes tout accidentelles : la mesure d'une grandeur est donc assimilable au tirage d'une boule dans une urne. Enfin, les tailles d'un groupe étendu d'hommes se répartissent de même très régulièrement de part et d'autre d'une taille moyenne, comme plusieurs mesures ou reproductions d'un même objet : elles peuvent donc être considérées comme les résultats d'autant d'essais plus ou moins approximatifs en vue de copier un type. Et il en est de même de beaucoup de caractères physiques de l'homme et des animaux. En ce sens, la loi de probabilités expliquerait des régularités qu'on relève dans le domaine des faits vitaux ${ }^{81}$.

Le conditionnel utilisé dans la dernière phrase laisse entrevoir la faille qu'Halbwachs estime identifier dans le raisonnement de Quetelet. En effet, «puisque Quetelet invoque les lois du hasard, pour expliquer que les résultats moyens se produiront le plus souvent, et que les résultats qui s'écarteront de la moyenne se disposeront de part et d'autre de celle-ci de façon très régulière, il faut nous demander à quelles conditions ces conditions joueront et, en particulier, comment ces conditions se peuvent réaliser dans le domaine de la vie ${ }^{82}$. Halbwachs estime justement que ces conditions ne sont pas remplies dans ce domaine, c'est-à-dire dans le domaine des sciences sociales et humaines: d'une part parce qu'il n'est pas possible d'extraire de leur contexte les événements humains puisque les hommes se situent dans une société et se définissent par rapport à elle; d'autre part parce que ces événements ne sont pas indépendants les uns des autres dans la mesure où les hommes dépendent les uns des autres et interagissent les uns avec autres.

Comment, dans ce contexte, la « loi des grands nombres », encore appelée «théorie des erreurs», pourrait-elle s'appliquer puisqu'elle suppose une parfaite indépendance entre les événements ou les causes qu'elle est censée régir. Même l'application de cette loi à l'anthropométrie lui semble abusive : «l'organisation sociale agit de façon [très] étendue : si les hommes vivaient en groupes absolument isolés [...], les différences de taille moyenne qui existaient de groupe à groupe iraient sans cesse en se consolidant ${ }^{83}$.

La loi des grands nombres ne s'applique pas car, estime-t-il, la régularité a une cause sociale et non probabiliste: la régularité constatée par le

\footnotetext{
${ }^{81}$ Ibid., 41-42.

${ }^{82}$ Ibid., 45.

${ }^{83}$ Ibid., 68 .
} 
sociologue dans ses études des faits sociaux n'est pas le résultat d'un jeu aléatoire de causes naturelles qui se compensent plus ou moins exactement, mais le produit d'une action normalisatrice de la société. Les forces qui poussent ou qui retiennent les individus ne sont pas indépendantes les unes des autres, et ne s'annulent pas mutuellement (en moyenne) comme le font les erreurs de mesure en physique. Il remet donc en cause, dans le cadre précis des études de la société et des phénomènes sociaux, la validité de la théorie des erreurs élaborée au XIX ${ }^{\mathrm{ème}}$ siècle par Laplace et Gauss et appliquée aux faits humains et moraux par Quetelet ${ }^{84}$. Sur ce point, Halbwachs se démarque de la pensée de Durkheim qui, dans les Règles de la méthode sociologique, affirme que si «la statistique fournit le moyen d'isoler» les faits sociaux «des formes qu'ils prennent dans les cas particuliers » c'est parce que «les circonstances individuelles qui peuvent avoir quelque part dans la production du phénomène s'y neutralisent mutuellement et, par suite, ne contribuent pas à le déterminer ${ }^{85}$. En revanche, Halbwachs est très proche de la pensée de Simiand sur ce point puisque ce dernier, comme lui-même, estime que «les régularités, les stabilités, les lois ne résultent pas de la combinaison de multiples causes constantes avec des causes variables et indépendantes les unes des autres ${ }^{86}$. Comme Simiand, il estime que ces régularités sont la manifestation du social, du collectif, de la cohésion des phénomènes sociaux. Elles sont même les preuves de l'existence du social, donc les justifications de la sociologie.

\section{$X$ - Sa conception de l'enquête empirique}

Son rejet de la loi des grands nombres ou, en d'autres termes, de l'hypothèse d'annulation des erreurs liées à des causes aléatoires, a une conséquence pratique importante: il interdit toute idée d'enquête qu'Halbwachs qualifie d'extensive (terme dont l'usage peut aujourd'hui surprendre dans ce contexte), c'est-à-dire toute enquête reposant sur l'observation d'un très grand nombre de cas simples. Ce type d'enquête suppose implicitement qu'en multipliant «les expériences, [il y a] plus de chances d'annuler les erreurs ou d'effacer l'influence des cas exceptionnels ${ }^{87}$. Plus précisément, «les enquêteurs qui ont appliqué la méthode extensive en même temps que celle du questionnaire ont bien eu l'impression des erreurs, des inexactitudes et des outils qui ne pouvaient pas

\footnotetext{
${ }^{84}$ Sur l'histoire de la loi des erreurs, la littérature est très abondante. Signalons par exemple ARMATTE (1991a). Sur la place de Quetelet dans cette histoire, voir ARMATTE (1991b).

${ }^{85}$ DURKHEIM, 1895, 9-10.

${ }^{86}$ LÉCUYER, 1991 ; voir aussi BOUVIER, 1977.

${ }^{87}$ HALBWACHS, 1912a, 151.
} 
manquer d'entrer dans les réponses des ouvriers interrogés, et ils ont cru qu'en multipliant les cas, ils obtiendraient, par jeu de la loi des grands nombres, une compensation et une atténuation croissante de ces fautes $»{ }^{88}$.

Pour des raisons semblables, Halbwachs rejette également la méthode leplaysienne qu'il qualifie d'intensive et qui consiste à «trouver un petit nombre de cas typiques et de les observer de très près $»{ }^{89}$. Toute la question est d'arriver à déterminer ce qui constitue un cas typique : ainsi, pour étudier les familles ouvrières et leurs budgets, «il ne peut être question d'exclure les familles qui dépensent trop, ou qui ne dépensent pas assez, soit au total, soit pour certains articles : ces expressions trop, ou pas assez, sont trop vagues, et ne peuvent déterminer que si on possède déjà un grand nombre de points de comparaison ${ }^{90}$. En ne s'attachant qu'aux cas jugés moyens, «on risque de tronquer la réalité ». Si Le Play parvient à identifier des familles typiques, «c'est parce que son expérience, ses connaissances sociologiques générales l'ont déjà conduit à se faire une idée des principales formes de la famille dans les sociétés contemporaines.[...] Il choisit une famille type comme un guide expérimenté vous conduit tout de suite à l'endroit d'où l'on découvre le mieux le pays ${ }^{91}$. Si ses connaissances a priori ne sont pas acquises, la méthode intensive n'est pas utilisable: c'est la situation dans laquelle Halbwachs estime se trouver : «nous n'avons aucune idée précise préalable des lois auxquelles obéissent les dépenses [...] Comment connaître du dehors les dépenses? $\gg^{92}$.

À ses yeux, la connaissance statistique n'est en rien synonyme de connaissance partielle, issue d'un échantillon de la population totale. S'inspirant à nouveau de la pensée de Simiand, il souligne que la recherche statistique doit porter uniquement sur des ensembles connus de façon exhaustive, systématique et complète, dont le dénombrement est « intégral ». En particulier, pour étudier un fait social évoluant dans le temps : «il faut étudier le phénomène en le suivant de façon ininterrompue, de son commencement jusqu'à son terme, de même qu'un botaniste ne s'en tiendra pas à observer une plante tous les premiers du mois, ou tous les dimanches, mais la suivra jour par jour, de la germination à la floraison ${ }^{93}$. Les statistiques ne rendant compte de la réalité sociale qu'à intervalles trop espacés ne permettent pas toujours de saisir la réalité des évolutions : «[Le statisticien] recense, par exemple, la population tous les cinq ans, tous les dix ans, [...] alors que c'est peut-être à une des années intermédiaires que

\footnotetext{
${ }^{88}$ Ibid., 152.

${ }^{89}$ Ibid., 151.

${ }^{90}$ Ibid., 159.

${ }^{91}$ Ibid., 158-159.

${ }^{92}$ Ibid., 159.

${ }^{93}$ HALBWACHS, 1944 (1935), 117.
} 
commence ou que finit un mouvement continu, que se produit une variation essentielle ${ }^{94}$. Dans son ouvrage de 1912 sur les dépenses et besoins de la classe ouvrière, il se félicite de ce que la durée d'observation et d'enregistrement des budgets ouvriers soit de un an ${ }^{95}$. Il n'imagine pas pouvoir conduire une étude sérieuse à partir d'enregistrements plus épisodiques : il ne suffit pas de « recueillir chaque année, ou à des intervalles assez rapprochés, des budgets tenus par des ménages de même profession et de même composition, par exemple, si l'on veut observer l'influence que les conditions économiques et leur évolution ont eue sur leurs dépenses. Car il se peut que les familles nouvellement étudiées ne se rattachent par aucun lien continu aux familles anciennes, que celles-ci aient disparu ou émigré, que celles-là soient arrivées depuis peu dans ce pays ou dans ce groupe ${ }^{96}$.

La seule méthode acceptable est donc la méthode cherchant à atteindre l'exhaustivité sur un grand nombre de situations, en tout cas un nombre plus élevé que les études monographiques. Les données qu'il utilise en 1912 semblent correspondre à cette exigence: «nos enquêtes allemandes ne soulèvent point les mêmes objections. D'une part, moins nombreux sans doute que les budgets recueillis par les enquêteurs américains et anglais, ceux qu'elles ont obtenus sont du moins des budgets véritables, des comptes tenus jour par jour d'un bout de l'année à l'autre, non des réponses vagues à des questions sommaires. D'autre part, moins complets que les budgets de Le Play et de ses disciples, ils ne se rapportent point à quelques ménages plus ou moins arbitrairement retenus comme typiques $\gg{ }^{97}$.

Remarquons que la question de la représentativité, comme celle du sondage, ne se posent pas à Halbwachs : en cela, il ne se démarque pas de son époque puisque dans les enquêtes statistiques de la première moitié de notre siècle, même celles conduites par la SGF, ces questions ne sont pas soulevées. En fait, ces questions ne sont pas encore à l'esprit des statisticiens de l'époque; et lorsqu'elles le sont, elles ne reçoivent pas de réponses pratiques. Il faut attendre les années 1940-1950 pour voir émerger ces questions et leurs réponses dans les enquêtes sociales et les données sociologiques ${ }^{98}$.

\footnotetext{
${ }^{94} \mathrm{Ibid}$.

${ }^{95}$ HALBWACHS, 1912a, 142-151.

${ }^{96}$ Ibid., 143 .

${ }^{97}$ Ibid., 160-161.

${ }^{98}$ DESROSIÈres, 1993, chap. 7.
} 


\section{XI - Le cadre théorique de son usage des statistiques}

Un des principes directeurs de son œuvre est la description objective de la réalité sociale qu'il conçoit comme multiforme et parfois contradictoire. C'est en tout cas le principe qui guide ses recherches de morphologie sociale. Une des formes concrètes que prend ce principe général est l'étude des classes sociales : Quelles sont leurs origines, leurs caractéristiques ? Qu'estce qui assure leur existence et leur cohésion? Comment et dans quelles circonstances les individus constituent-ils un groupe social ou une classe sociale? Dans quelle mesure l'appartenance à une catégorie sociale détermine-t-elle les conduites sociales? Ces questions traversent toute l'œuvre d'Halbwachs, jusqu'à son texte Mémoire et société (1948) publié de façon posthume. Les réponses qu'il apporte à cette question évoluent au fil de ses écrits mais plusieurs grandes lignes persistent: l'individu et le groupe interagissent ; l'individu, sa conscience et sa mémoire, sont enracinés dans le groupe social ou la classe sociale d'appartenance ; les actions quotidiennes de chaque individu sont contraintes par son groupe ou sa classe ; et inversement l'individu contribue à définir le groupe, la mémoire et la conscience collectives. Ces principes constituent ce qu'il est possible d'appeler « l'hypothèse de réciprocité généralisée entre l'individu et le groupe » ${ }^{99}$.

Comment ces réflexions sur la morphologie sociale et les groupes sociaux s'articulent-elles avec ces usages et ses conceptions de la statistique ? Cette question, simple dans son énoncé, renvoie au moins à quatre types de réponses. À un premier niveau pratique, dans ses études de morphologie des groupes sociaux, Halbwachs conçoit les données statistiques et leurs interprétations comme des moyens de connaissance des groupes et de leurs comportements : c'est dans ce but qu'il a recours à des données statistiques abondantes pour saisir les causes du suicide, les budgets ou les besoins des ouvriers, les budgets familiaux français ou américains. La statistique permet de parfaitement remplir l'objectif général qu'il assigne à ses études sociologiques puisqu'elles permettent de saisir «dans quelle mesure la détermination des dépenses dans des groupes de ménages permet [...] de reconnaître d'abord les rapports qui existent entre la répartition des dépenses et le niveau de revenu, et la grandeur de la famille et aussi, au-delà, quelle influence peut exercer, à cet égard, le fait d'appartenir à une classe définie : classe des employés, ou classe des ouvriers ${ }^{100}$. Le recours à des données quantitatives n'a toutefois pas pour objectif de quantifier les niveaux de vie ou les causes de suicide: il s'agit simplement d'avoir des indicateurs objectifs pour saisir qualitativement, c'est-à-dire dans leurs formes et

\footnotetext{
${ }^{99}$ NAMER, 1997, 14.

${ }^{100}$ HaLBWACHS, 1933, Budget des familles aux États-Unis et en Allemagne, Bulletin de l'Institut Français de Sociologie, 52 ; cité dans MARCEL, 1997, 209.
} 
structures, les différents niveaux ou modes de vie. Le recours à des données quantitatives ne permet pas de connaître la «nature » des événements sociaux mais simplement « une matière pour la réflexion; matière en tant que trace directe et immédiatement quantifiée ${ }^{101}$ de ces événements. À un second niveau pratique, les statistiques sur les «faits de population » lui permettent de saisir le cadre démographique général de la vie sociale : il ne les étudient pas, comme nous l'avons déjà signalé, pour eux-mêmes mais seulement dans le but de saisir les contraintes « matérielles » pesant sur la vie sociale.

Ensuite, à un premier niveau théorique ou épistémologique, la statistique constitue pour lui le seul instrument permettant de saisir ce qui caractérise, non pas un individu, mais un groupe social : la statistique permet d'atteindre les dimensions collectives, les propriétés des classes sociales. Sans elle, seules les dimensions individuelles peuvent être connues, ce qui est évidemment insuffisant aux yeux d'Halbwachs. Enfin, à un second niveau théorique, la conception de la statistique rejoint chez Halbwachs sa conception de la diversité et de l'hétérogénéité des groupes sociaux : il ne conçoit pas le calcul de la moyenne comme permettant de saisir les caractéristiques de l'individu «moyen » c'est-à-dire de l'individu idéal dont les autres ne seraient que des « approximations » mais comme permettant de saisir les traits du collectif, entité ayant une existence et des caractéristiques propres non réductibles à celles des individus: la statistique permet « d'atteindre les caractères d'un groupe, qui ont une réalité dans le groupe tout entier, mais qu'on ne découvrirait dans aucun membre de ce groupe pris à part et isolément » ${ }^{102}$. S'il faut supposer l'existence d'une « espèce » pour comprendre le maintien de l'intégrité des phénomènes sociaux, cette espèce est à situer dans le groupe et non dans l'individu : Halbwachs conçoit cette « espèce sociale » comme «l'ensemble des tendances collectives qui assurent la subsistance d'une organisation sociale ${ }^{103}$, comme « tout un ensemble de forces générales, antérieures et extérieures et, en tout cas, qui n'ont point leur origine dans sa nature individuelle ${ }^{104}$. Tous les «individus dépendent de [cette] espèce, qui les a précédés de même qu'elle les enveloppe ${ }^{105}$.

\section{Conclusion : qu'est-il advenu d'Halbwachs et de sa pensée ?}

Maurice Halbwachs est, avec Simiand, le durkheimien qui s'est le plus intéressé à la statistique, à l'économie mathématique et à la démographie.

\footnotetext{
${ }^{101}$ ALEXANDRE, 1940-1948, 5.

${ }^{102}$ HalBWACHS, 1944 (1935), 114.

${ }^{103}$ HaLBWACHS, 1912b, 150.

${ }^{104}$ Ibid., 165.

${ }^{105}$ Ibid., 164.
} 
Mais, en même temps, il est celui qui a développé une pensée critique forte et systématique vis-à-vis des représentations produites par les approches quantitatives et mathématisées de la réalité sociale : il a refusé l'abstraction mathématique; il a toujours considéré que les faits démographiques ou économiques ne peuvent pas être étudiés pour eux-mêmes ou en eux-mêmes et que leur analyse nécessite de les replacer de façon précise dans leur contexte social ; il a critiqué l'hypothèse d'autonomie des faits nécessaire aux mathématiques de son époque ; il a rejeté la mise en équation mathématique et les fictions des graphiques excessivement simplificateurs; il a dénoncé le concept de moyenne lorsqu'il est utilisé pour élaborer une théorie... Et en même temps, il a côtoyé nombre de statisticiens et de mathématiciens en raison de ses responsabilités au sein d'organisations statistiques: l'Institut International de Statistique, la Statistique Générale de France, la Société Statistique de Paris. Et n'a-t-il pas rédigé, avec Alfred Sauvy, jeune polytechnicien entré à la SGF en 1923, l'article consacré à la démographie dans la grande Encyclopédie Française de Lucien Febvre ${ }^{106}$ ? Et n'a-t-il pas écrit avec un grand mathématicien un manuel de probabilités ? Cette identité ambivalente fait de lui un proche de la communauté des statisticiens sans en faire un membre à part entière : utilisateur et penseur des statistiques, il est aussi critique vis-à-vis des usages qui en sont fait couramment.

Cette ambivalence dans son statut se double d'une ambivalence dans sa pensée du pouvoir des statistiques. Cherchant à penser le tout (le groupe, la classe, la société) comme irréductible à la fois à la simple collection de ses membres et à la simple accumulation de causes indépendantes les unes des autres, Halbwachs cherche à identifier les formes d'analyse statistique compatibles avec ces deux nécessités. Cela le conduit à refuser les hypothèses de Quetelet et, plus généralement, à refuser toute hypothèse considérant l'individu comme un représentant d'un unique «type normal» : il n'imagine pas que la moyenne permette d'identifier ce «type normal». Cela le conduit également à refuser toute décomposition des causes en causes élémentaires, en causes sociales simples ou hiérarchisées ; à décomposer ce qu'il appelle les « systèmes d'influences ». Il reconnaît par exemple, dans son étude du suicide, qu'il est impossible d'étudier séparément l'influence de la religion et celle des facteurs comme la situation domestique, politique, économique ou géographique. Chaque pas qu'il fait en direction d'une décomposition des causes, est contrebalancé par un propos théorique estimant que ce pas peut l'amener trop loin, c'est-à-dire l'éloigner de la réalité sociale et lui faire construire des mondes abstraits, fictifs. Balancé entre son désir d'identifier les causes sociales et sa crainte que cette identification le conduise à décomposer artificiellement la réalité sociale, Halbwachs est parfois gêné : sa pensée lui interdit de faire ce que les outils statistiques lui

\footnotetext{
${ }^{106}$ HALBWACHS, 1936 b.
} 
autorisent. Saisir le tout en le décomposant, sans prétendre réduire ce tout à ces seuls éléments, est chose impossible. S'il n'est pas totalement paralysé, il est parfaitement conscient de cette contrainte : «il n'est pas possible, jusqu'à présent, d'isoler le facteur religieux et de mesurer son action. C'est un problème qui demeure posé, et l'on n'entrevoit même pas comment on pourrait le résoudre ${ }^{107}$. En somme, tout en attribuant un rôle technique central à la science statistique, il refuse de donner trop de pouvoir à cette technique : ne pas utiliser la statistique c'est ne pas se donner les moyens de saisir le collectif et d'étudier positivement la société ; utiliser la statistique c'est risquer de mener des analyses artificielles, éloignées de la réalité, en se laissant absorber par une logique toute mathématique. Sa position est donc délicate: faire appel à la statistique pour étudier des faits sociaux, sans réduire ces derniers à de simples «événements statistiques » (c'est-à-dire saisis individuellement, comparés puis assimilés).

Cette double ambivalence, statutaire et intellectuelle, lui a certainement valu de disparaître rapidement de l'esprit des statisticiens d'après-guerre. Alfred Sauvy, qui avait pourtant travaillé avec lui, déclarera plus tard qu'Halbwachs n'était pas un véritable statisticien : s'il dit «avoir été en très bons termes avec Halbwachs » c'est pour immédiatement ajouter qu'il « contestait ses connaissances statistiques ». Un autre membre de la SGF, Henry Bunle, fait entendre un son de cloche identique : Halbwachs «était un littéraire » et non pas « un scientifique au sens où nous l'entendons nous ${ }^{108}$. Et dans une conférence de 1949 consacrée à la théorie de l'homme moyen de Quetelet, son ancien collègue et collaborateur Maurice Fréchet ne le cite pas, n'évoque même pas sa mémoire ${ }^{109}$.

Cette double ambivalence peut également contribuer à expliquer le relatif mais réel oubli dans lequel Halbwachs est tombé après-guerre chez les sociologues : il sera peu cité après-guerre ${ }^{110}$. Il n'est au fond, qu'une des victimes de la «disgrâce » de la pensée sociologique française de l'entredeux-guerres. Et si, comme Simiand ou Mauss, Halbwachs est parfois cité, il l'est davantage à titre symbolique ou emblématique, qu'à titre purement intellectuel : l'homme et ce qu'il représentait ne sont pas totalement oubliés, mais sa pensée n'est plus utilisée et son œuvre n'est pas réellement poursuivie. C'est ce que certains nomment le «drame» ${ }^{111}$ de l'école française de sociologie. De ce point de vue, Halbwachs, si soucieux de la

\footnotetext{
${ }^{107}$ HALBWACHS, 1930, 8.

${ }^{108}$ Interviews d'A. Sauvy et d'H. Bunle par Alain Desrosières : DESROSIÈRES, 1982, 165.

${ }^{109}$ FRÉCHET, 1949.

${ }^{110}$ VANNIER, 1999, 571.

${ }^{111}$ MARCEL, 1997, 120-124. François Simiand sera une autre des victimes, et peut-être la principale, de cet oubli : après-guerre aucune recherche ne s'inspirera directement de ses travaux.
} 
condition et de l'avenir de la sociologie, aurait sans doute été déçu s'il avait survécu à son « assassinat » ${ }^{112}$ en 1945.

Pourtant, si Halbwachs n'a pas réussi à penser les processus stochastiques (comme le souligne justement Lazarsfeld, qui est par ailleurs sévère à son encontre ${ }^{113}$ ) c'est-à-dire l'idée qu'il est possible de formaliser mathématiquement la dépendance entre des événements, il n’en a pas moins introduit l'idée, peu présente chez les statisticiens de son temps, que les phénomènes sociaux interagissent les uns sur les autres, que les individus entretiennent des liens qui font et défont leurs actions; il a également fortement contribué à introduire une distinction entre les mesures répétées d'un même objet, et les mesures d'objets semblables mais distincts. Il a ainsi favorisé l'abandon de l'idée, très laplacienne et quetelesienne, que les mesures statistiques de caractéristiques collectives pouvaient être interprétées comme des mesures répétées de caractéristiques individuelles, et qu'elles pouvaient donc être supposées indépendantes. Cette idée, très prégnante dans toutes les sciences du XIX ${ }^{\text {ème }}$ siècle, a marqué les sciences humaines et sociales naissantes, et notamment la psychologie, l'économie et la sociologie ${ }^{114}$. Halbwachs a incontestablement concouru à faire évoluer la pensée statistique en sciences sociales.

En cela, il se situe dignement dans la tradition qui, depuis les enquêtes sociales et démographiques du XIX ${ }^{\text {ème }}$ siècle, à travers Quetelet, Villermé, Parent du Châtelet, relie les instances statistiques et les responsables des enquêtes statistiques, aux spécialistes des sciences morales et sociales naissances ${ }^{115}$. Cherchant, dans l'entre-deux-guerres, à saisir statistiquement les réalités sociales pour déduire des données statistiques une intelligence et une compréhension du social (voire les moyens de l'action politique et sociale), Halbwachs incarne parfaitement, en compagnie de Simiand, ce lien. Certaines institutions contemporaines incontournables, comme l'INSEE et l'INED, sont aujourd'hui les héritières de cette tradition dont Halbwachs a été un maillon essentiel bien que largement oublié.

Olivier MARTIN

Université Paris $V$ - CERLIS

12, rue Cujas - F-75230 Paris Cedex 05

E-mail :olmartin@citi2.fr

\footnotetext{
${ }^{112}$ Nous empruntons le terme «d'assassinat» à l'article de Pierre Bourdieu publié sous le titre «L'assassinat de Maurice Halbwachs » (BOURDIEU, 1987); article d'ailleurs fortement inspiré de CRAIG (1979).

${ }^{113}$ LAZARSFELD, 1970, 115-118

${ }^{114}$ Sur la psychologie, voir MARTIN (1997) ; sur l'économie, voir ARMATTE (1995). Et plus généralement, se reporter aux deux volumes de The Probabilistic Revolution (KRÜGER et al., 1987).

${ }^{115}$ MARTIN, 1998.
} 
Olivier MARTIN

\section{Bibliographie}

AlEXANDRE J., 1940-1948, Maurice Halbwachs, Année sociologique, Troisième série, 1, 3-10.

ARMATte M., 1991a, Théorie des erreurs, moyenne et loi normale, in Feldman J., Lagneau G., Matalon B., 1991, 63-84.

ARMATTE M., 1991b, La moyenne à travers les traités de statistique au XIX $^{\text {ème }}$ siècle, in Feldman J., LAGneau G., Matalon B., 1991, 85-106.

ARMATte M., 1995, Histoire du modèle linéaire: formes et usages en statistique et économétrie, polycopié, thèse de doctorat de l'EHESS.

Baudelot C., Establet R., 1994, Maurice Halbwachs. Consommation et société, Paris, PUF.

BESNARD P., 1979, La formation de l'équipe de l'Année sociologique, Revue française de sociologie, XX, 7-31.

Bourdieu P., 1987, L'assassinat de Maurice Halbwachs, La liberté de l'esprit, 16, 161-168.

BouviER J., 1977, François Simiand, la statistique et les sciences humaines, in Pour une histoire de la statistique, Paris, INSEE, tome 1, 431-443.

CRAIG J.E., 1979, Maurice Halbwachs à Strasbourg, Revue française de sociologie, XX, 273-292.

DESROSIÈRES A., 1982, Un essai de mise en relation des histoires récentes de la statistique en sociologie, in Actes de la journée d'étude "Sociologie et Statistique » (octobre 1982), Paris, INSEE-SFS, 161-182.

DeSROsiÈres A., 1991, Masses, individus, moyennes : la statistique sociale au XIX ${ }^{\text {ème }}$ siècle, in Feldman J., LAgneAU G., MAtalon B., 1991, 245273.

DesrosiÈres A., 1993, La politique des grands nombres. Histoire de la raison statistique, Paris, La Découverte.

DURKHEIM E., 1895, Les règles de la méthode sociologique, Paris, PUF.

Feldman J., Lagneau G., Matalon B., Éds., 1991, Moyenne, milieu, centre. Histoire et usages, Paris, Éditions de l'École des Hautes Études en Sciences Sociales.

FOURNIER M., 1994, Marcel Mauss, Paris, Fayard.

FRÉCHET M., 1949, Réhabilitation de la notion statistique de l'homme moyen, Paris, les conférences du Palais de la découverte.

FRÉCHet M., HALBWACHS M., 1924, Le calcul des probabilités à la portée de tous, Paris, Dunod.

GEMELli G., 1998, Paul Lazarsfeld et la France au milieu des années soixante, in LAUTMAN J., LECUYER B.-P. (Eds.), Paul Lazarsfeld (19011976). La sociologie de Vienne à New-York, Paris, l'Harmattan, 465-501. 
HALBWACHS M., 1912a, La classe ouvrière et les niveaux de vie. Recherches sur la hiérarchie des besoins dans les sociétés industrielles contemporaines, Paris, Alcan.

HaLBWACHS M., 1912b, La théorie de l'homme moyen. Essai sur Quetelet et la statistique morale, Paris, Alcan.

HALBWACHS M., 1923, L'expérimentation statistique et les probabilités, Revue philosophique, 96, 340-371 (repr. in HALBWACHS, 1972, 275-307).

HaLbWaChS M., 1923-1924, Questions de méthodologie: La statistique, Année sociologique, Nouvelle série, I, 279-283.

HALBWACHS M., 1929, Méthodes statistiques, Annales d'histoire économique et sociale, 1, 423-428.

HalbWachs M., 1930, Les causes du suicide, Paris, Félix Alcan.

HalbwaChs M., 1931, La comparaison des méthodes statistiques, Annales d'histoire économique et sociale, 3, 72.

HalBWACHS M., 1932, Titres et Travaux, s.l.n.ed.

HALBWACHS M., 1933, L'évolution des besoins dans les classes ouvrières, Paris, Librairie Félix Alcan.

Halbwachs M., 1935, De la statistique morale en général, Annales sociologiques, série C, fascicule 1, 169.

HalbwachS M., 1936a, La méthodologie de François Simiand. Un empirisme rationaliste, Revue philosophique, 121, 281-319 (repr. in HALBWACHS, 1972, 349-389)

HaLBWACHS M., 1936b, Le point de vue du nombre, in L'encyclopédie française, Paris, Librairie Larousse, tome VII.

Halbwachs M., 1937a, Les classes sociales, Paris, Centre de documentation universitaire (Les cours de la Sorbonne).

HALBWACHS M., 1937b, Note sur l'emploi des coefficients de corrélation ou de co-variation principalement en sociologie, Annales sociologiques, C, fascicule 2, 141-144.

HalbWaChS M., 1937c, Le point de vue du sociologue, X-Crise Bulletin, 34, 23-30 (republié dans HaLBWACHS, 1972, 390-408)

Halbwachs M., 1938, Morphologie sociale, Paris, Armand Colin.

HALBWACHS M., 1939, Les causes de mort en cartogrammes, Annales d'histoire sociale, 11, 313-314.

HALBWACHS M., 1944 (1935), La statistique en sociologie, in C.I.S. (CENTRE INTERNATIONAL DE SYNTHÈSE), La statistique. Ses applications. Les problèmes qu'elles soulèvent (septième semaine de synthèse, 3-8 juin 1935), Paris, PUF, 113-160.

HalbWaCHS M., 1972, Classes sociales et morphologie (textes réunis par Victor Karady), Paris, Minuit.

Karady V., 1972, Biographie de Maurice Halbwachs, in Halbwachs M., Classes sociales et morphologie, Paris, Minuit, 7-22.

KRÜGER L. et al., 1987, The Probabilistic Revolution: Volume 1 : Ideas in History; Volume 2 : Ideas in Sciences, Cambridge, MIT Press. 
LAZARSFELD P., 1970 (1961), Notes sur l'histoire de la quantification en sociologie: les sources, les tendances, les grands problèmes, in Philosophie des sciences sociales, Paris, Gallimard.

LECUYER B.-P., 1991, Singularité des faits et vérités statistiques : à partir de la controverse Simiand-Seignobos, in Feldman J., LAGNEAU G., MATALON B., 1991, 275-287.

Le Gall P., 1994, Histoire de l'économétrie (1914-1944) : l'érosion du déterminisme, polycopié, thèse de doctorat de l'Université Paris I.

LENOIR R., 1997, Halbwachs sociologue ou démographe?, in de Montlibert Ch., Maurice Halbwachs 1977-1945, Strasbourg, Presses Universitaires de Strasbourg, 47-61.

MARCEL J.-C., 1997, Les avatars de l'héritage durkheimien. Une histoire de la sociologie en France (1920-1958), polycopié, thèse de doctorat de l'Université Paris I.

MARTIN O., 1997, La mesure de l'esprit. Origines et développements de la psychométrie (1900-1950), Paris, l'Harmattan.

MARTIN O., 1998, Du sujet à l'individu social : l'émergence des statistiques sociales et morales (XVII ${ }^{\text {ème }}$-XIX ${ }^{\text {ème }}$ siècles), Tumulte, 10, 53-77.

Montlibert de Ch., 1997, Maurice Halbwachs et les classes sociales, in de Montlibert Ch., Maurice Halbwachs 1977-1945, Strasbourg, Presses Universitaires de Strasbourg, 63-74.

MuCChiElli L., 1998, La découverte du social. Naissance de la sociologie en France (1870-1914), Paris, La Découverte.

NAMER G., 1997, Halbwachs, in MONTLIBERT de Ch., Maurice Halbwachs 1977-1945, Strasbourg, Presses Universitaires de Strasbourg, 11-16.

PFEFFERKORN R., 1997, Maurice Halbwachs et l'économie politique, in MontLiBert de Ch., Maurice Halbwachs 1977-1945, Strasbourg, Presses Universitaires de Strasbourg, 31-46.

Simiand F., 1922, Statistique et expérience. Remarques de méthode, Paris, Marcel Rivière.

SimiAnd F., 1987, Méthode historique et sciences sociales (textes choisis et présentés par M. Cedronio), Paris, Éditions des archives contemporaines.

VANNIER P., 1999, Un laboratoire pour la sociologie? Le Centre d'études sociologiques (1946-1968) ou Les débuts de la recherche sociologique en France, polycopié, thèse de doctorat de l'Université Paris V-René Descartes.

VERRET M., 1972, Halbwachs ou le deuxième âge du durkheimisme, Cahiers internationaux de sociologie, LIII, 311-336. 
Revue d'histoire des sciences humaines 